\title{
A Unit Commitment Model with Implicit Reserve Constraint Based on an Improved Artificial Fish Swarm Algorithm
}

\author{
Wei Han, Hong-hua Wang, Xin-song Zhang, and Ling Chen \\ College of Energy \& Electrical Engineering, Hohai University, Nanjing 211100, China \\ Correspondence should be addressed to Wei Han; hanwei860610@126.com
}

Received 8 June 2013; Revised 29 September 2013; Accepted 1 November 2013

Academic Editor: Pedro Ribeiro

Copyright ( 2013 Wei Han et al. This is an open access article distributed under the Creative Commons Attribution License, which permits unrestricted use, distribution, and reproduction in any medium, provided the original work is properly cited.

\begin{abstract}
An implicit reserve constraint unit commitment (IRCUC) model is presented in this paper. Different from the traditional unit commitment (UC) model, the constraint of spinning reserve is not given explicitly but implicitly in a trade-off between the production cost and the outage loss. An analytical method is applied to evaluate the reliability of UC solutions and to estimate the outage loss. The stochastic failures of generating units and uncertainties of load demands are considered while assessing the reliability. The artificial fish swarm algorithm (AFSA) is employed to solve this proposed model. In addition to the regular operation, a mutation operator (MO) is designed to enhance the searching performance of the algorithm. The feasibility of the proposed method is demonstrated from 10 to 100 units system, and the testing results are compared with those obtained by genetic algorithm (GA), particle swarm optimization (PSO), and ant colony optimization (ACO) in terms of total production cost and computational time. The simulation results show that the proposed method is capable of obtaining higher quality solutions.
\end{abstract}

\section{Introduction}

During the operation of a power system, the balance of active power is often interrupted by some unpredictable factors, such as a random load change, transmission and transformation of power equipment, and failure of the generating unit. In order to keep the balance of power system, a reasonable amount of spinning reserve needs to be set in advance. During the actual power generation dispatch, the system spinning reserve requirements are predetermined by the knowledge of experienced power system operators and introduced as a set of operating constraints which give better performance of the power system [1,2]. The basic task of the unit commitment (UC) model containing the spinning reserve constraint is to schedule the planning of power generation and the arranging output with minimum cost in order to satisfy the power load, spinning reserve, and operational condition of units [3].

To deal with the problem of random failure of the unit, the reserve demand was defined as the largest capacity of the installed generating units within the system. Furthermore, some practical systems for scheduling would assume the spinning reserve to be a fixed percentage of power loads. The selecting principle of deterministic reserve neither considered the various random factors of the operating system nor provided the cost of reserve. Therefore, the system state would be subjective to the experience of the dispatcher and in some cases the system would not operate efficiently from economy aspect. As more and more largescale renewable energy sources are injected into the grid, the uncertainty of the power system will be significantly increased and the current calculation of the system spinning reserve would be more inefficient. Consequently, Anstine et al. [4] and Li and Zhou [5] put forth a principle of probabilistic reserve with the risk of UC model. Based on reliability constraints, Snyder et al. [6] proposed a UC model using the inequality constraints of reliability index to replace the deterministic reserve constraints of the traditional UC model; a good performance was obtained. However, a lot of subjectivity is introduced by selecting the threshold of probabilistic reliability index and the deterministic reserve.

Based on the above discussion, the spinning reserve constraint is removed from the UC model and an implicit reserve constraint unit commitment (IRCUC) model is proposed in 
this paper. In addition, the objective function of this model is extended from the minimal production cost of traditional UC model to the minimal sum of production cost and outage loss. That is, if the spinning reserve capacity is scheduled too much in the planning of power generation, then the outage loss would be reduced while the production cost would be increased; on the contrary, if the reserve capacity is scheduled too little, then the production cost would be decreased while the outage loss would be increased.

Same as the traditional UC model, the IRCUC model proposed in this paper is a combinatorial optimization problem with time and nonlinearity, which contains continuous and discrete variables, so it is difficult to get the analytical solution. To solve the UC problem, extensive researches have been carried out by domestic and foreign scholars. The solutions are mainly attributed to the following categories: (1) priority list (PL) algorithm [7, 8]; (2) mathematical algorithms, including branch-bound algorithm [9], bender's decoupling algorithm [10], Lagrange relaxation algorithm $[11,12]$, dynamic programming algorithm and mixed integer linear programming (MILP) algorithm [13]; (3) artificial intelligence algorithms, such as genetic algorithm (GA) [1416], particle swarm optimization (PSO) [17], ant colony optimization (ACO) [18], and simulated annealing (SA) [19].

In the solving process of IRCUC model, the reliability of generation schedule is evaluated repeatedly to estimate the power loss. Therefore, compared with the traditional UC model, the solving of IRCUC model will become more difficult. In this paper, a novel heuristic called artificial fish swarm algorithm (AFSA) is introduced into this topic for the first time [20]. This algorithm has an ability of optimizing, and it is found efficient and reliable in solving the UC problem. AFSA has many advantages, including good global convergence, strong robustness, insensitivity to initial values, simplicity in implementing, and so forth [21]. The proposed AFSA has been widely used in many different applications such as parameters analysis, neural network classifiers, signal processing, network combinatorial optimization, and complex function optimization [22-25]. For these reasons, this paper uses the AFSA to solve the IRCUC model. Different from the standard AFSA operations, an intelligent mutation operator (MO) is employed to promote the optimization performance of the algorithm. To verify the advantages of the improved AFSA method, the proposed method is tested and compared to other methods on the systems with the number of generating units from 10 to 100 . The simulation results demonstrate that the improved AFSA is superior to other methods in terms of total production cost and computational time.

The rest of this paper is organized as follows. Section 2 sets up the mathematical model of IRCUC in detail. Section 3 introduces a brief overview of reliability evaluation. Section 4 proposes the improved AFSA and applies it to the solving of IRCUC model. In addition, the model and the solution algorithm are examined and discussed through the utilization of different testing systems in Section 5. Finally, Section 6 draws the conclusion.

\section{Mathematical Model of IRCUC}

Under the satisfaction of the various conditions, the basic task of UC model is to seek the planning of power generation and the arranging output with minimum cost. The total production cost $C_{f}$ over the entire scheduling periods is the sum of the operating cost and start-up cost of the committed units. Thus, the UC objective function of minimum cost can be expressed as follows:

$$
C_{f}=\sum_{t=1}^{T} \sum_{i=1}^{M}\left[F_{i}\left(P_{i, t}\right) U_{i, t}+S_{i, t}\left(1-U_{i, t-1}\right) U_{i, t}\right],
$$

where $T$ is the dispatch period under consideration; $M$ is the number of generating units; $P_{i, t}$ is the power generation of unit $i$ at time $t$, which is the continuous variable for optimization; $U_{i, t}$ is the operating state of unit $i$ at time $t$, which is the discrete variable for optimization ( 1 if the unit is on and 0 if the unit is off); $C_{f}$ is the total cost of power generation, which is comprised of fuel cost and start-up cost and given by part 1 and part 2 of (1), respectively, $F_{i}\left(P_{i, t}\right)$ is the fuel cost of unit $i$ at time $t$, which is approximately expressed by the following quadratic function [9]:

$$
F_{i}\left(P_{i, t}\right)=a_{i}+b_{i} P_{i, t}+c_{i} P_{i, t}^{2},
$$

where $a_{i}, b_{i}$, and $c_{i}$ are the fuel cost coefficients of unit $i$, respectively. The start-up cost of unit $i$ at time $t S_{i, t}$ in (1) is given by the following exponential function:

$$
S_{i, t}= \begin{cases}S_{h i}, & T_{i, \text { off }} \in\left[T_{i, \text { down }}, T_{i, \text { down }}+T_{i, \text { cold }}\right], \\ S_{c i}, & T_{i, \text { off }} \in\left[T_{i, \text { down }}+T_{i, \text { cold }},+\infty\right],\end{cases}
$$

where $S_{h i}$ and $S_{c i}$ are the hot and the cold start-up cost of unit $i$, respectively; $T_{i, \text { off }}$ is the continuous off time of unit $i$ up to time $t ; T_{i \text {,down }}$ is the minimum down time of unit $i$; $T_{i \text {,cold }}$ is the cold start-up time of unit $i$.

The minimization of the objective function is subject to a number of system and generating unit constraints.

(1) Generation Limit Constraints. The power produced by each unit must be within certain limits and that is indicated by

$$
P_{i, \min } \leq P_{i, t} \leq P_{i, \max },
$$

where $P_{i, \max }$ and $P_{i, \min }$ are the maximum and minimum power output of unit $i$, respectively.

(2) Load Balance Constraints. The total generated power at each hour must be equal to the load of the corresponding time. This constraint is given by

$$
P_{l, t}=\sum_{i=1}^{M} P_{i, t} U_{i, t},
$$

where $P_{l, t}$ is the system demand at time $t$.

(3) Unit Minimum Uptime/Downtime Constraints. A unit must be on for a certain number of hours before it can be 
shut down. A unit must be off for a certain number of hours before it can be brought online. Consider

$$
\begin{gathered}
T_{i, \text { off }} \geq T_{i, \text { down }}, \\
T_{i, \text { on }} \geq T_{i, \text { up }},
\end{gathered}
$$

where $T_{i \text {,on }}$ is continuously on time of unit $i$ up to time $t$ and $T_{i \text {,up }}$ is minimum uptime of unit $i$ up to time $t$.

(4) Generation Ramping Constraints. The ramping rate constraints are activated, if a unit remains in operation for two successive hours. In that case,

$$
\Delta_{i, \min } \leq P_{i, t}-P_{i, t-1} \leq \Delta_{i, \max }
$$

where $\Delta_{i, \min }$ and $\Delta_{i, \max }$ represent the lower and upper ramping rate limits of the $i$ th unit, respectively.

(5) Spinning Reserve Constraints. As mentioned before, spinning reserve constraints can be taken into consideration by using either deterministic criteria or probabilistic techniques. These requirements can be specified in terms of excess megawatt capacity, which is expressed by

$$
P_{l, t}+R_{t} \leq \sum_{i=1}^{M} P_{i, \max } U_{i, t},
$$

where $R_{t}$ is the system spinning reserve requirement at time $t$, which depends on the operating experience by the dispatcher.

In order to ensure the reliability of power system, the demanded reserves of all time are predetermined by the dispatcher according to the operating experience and also are introduced into the UC model as constraint conditions. Actually, the demanded reserves are related to various factors. Therefore, it is not easy to predetermine the spinning reserve capacity of the power system. In general, the increased value of $R_{t}$ would result in the number of start-up units growing, thereby increasing the production cost. On the contrary, the decreased value of $R_{t}$ will reduce the reliability of the system and increase the outage cost. Therefore, this paper would remove the spinning reserve in (8) from the UC model and propose the IRCUC model which includes the implicit spinning reserve constraint. At the same time, the optimization model is extended from the minimal production cost of traditional UC model to the minimal sum of the production cost and the outage loss, namely,

$$
\mathrm{O}_{f}=C_{f}+\sum_{t=1}^{T} E_{t}\left(U_{i, t}\right) \times V_{\mathrm{oll}},
$$

where $O_{f}$ is the minimal sum of the production cost and the outage loss; $E_{t}$ is the expected energy not served (EENS) at time $t ; V_{\text {oll }}$ is the value of lost load, which can be obtained via the statistical survey of the users. It is needed to point out that due to ignoring the climbing constraints of the generating units, the value of $E_{t}$ is only associated with the start-up planning of time and has nothing to do with output power of each unit. That is to say, the reliability index EENS is the function of discrete variables $U_{i, t}$ and irrelevant to the continuous variables $P_{i, t}$.

It follows from (9) that the production cost and the outage loss are related to the system spinning reserve requirements. For this reason, the demand and reserve constraints are not explicitly given in the IRCUC model. Rather, the tradeoff between the production cost and the outage loss of the objective function is implicit.

\section{Reliability Evaluation of IRCUC}

The foundation of solving the IRCUC model is to evaluate the reliability of power generation. The reliability index calculated only depends on considering the load uncertainties and the random failures of units.

3.1. Reliability Model of Units. Two state models are adopted by the units, which include the normal state and the fault state. The IRCUC model in this paper is mainly used for short-term scheduling of the power system (generally less than 24 hours). The possibility of repairing or replacing the faulty units is ignored in such a short time [15]. Therefore, the probability of faulty unit $f_{i, t}$ is expressed by the outage replacement rate (ORR) of unit $i$ at time $t$. The index varies along with time and can be expressed by

$$
f_{i, t}=1-\exp \left(\lambda_{i} t\right) \approx \lambda_{i} t
$$

where $\lambda_{i}$ is the failure rate of unit $i$.

3.2. Load Fluctuation Model. In the IRCUC model, the load $P_{l, t}$ is the result of load forecasting at time $t$. The statistics show that the load forecasting result at a future moment can be regarded as a random variable and its uncertainty is approximately reflected by the normal distribution. $\mu_{P}$ and $\sigma_{P}$ are the mean and the standard deviation of the active load forecasting, respectively, $[26,27]$. Then, the probability density function of the active load $P_{l}$ is computed by

$$
f\left(P_{l}\right)=\frac{1}{\sqrt{2 \pi} \sigma_{P}} \exp \left[-\frac{\left(P_{l}-\mu_{P}\right)^{2}}{2 \sigma_{P}^{2}}\right] .
$$

Assuming the load power factor as a constant, then the reactive power load $Q_{l}$ varies with the $P_{l}$ by the power factor.

3.3. Calculation of the Reliability Index. For calculating the $E_{t}$, the following assumptions are needed.

(1) The random fluctuation of power load and the random failures of units are assumed to be the independent random events.

(2) Random failure of each unit is independent of other units. Considering that the probability of faulty unit is small, the multiple failures of units are ignored in this paper. In other words, there is one unit out of order at most in every moment.

It is supposed that there are $m$ units $(m \leq M)$ in startup state at time $t$. Taking the random failures of units into 
consideration, the available generating capacity of this period $G_{t}$ is a discrete random variable, and the probability density function is expressed as follows:

$$
P\left\{G_{t}=G_{j}\right\}=p_{j}, \quad j=0,2, \ldots, m,
$$

where $G_{0}$ is the available generating capacity of $m$ units under the normal condition and $p_{0}$ is the probability corresponding to the event, which can be calculated by

$$
\begin{gathered}
G_{0}=\sum_{j=1}^{m} P_{j, \max }, \\
p_{0}=\prod_{j=1}^{m}\left(1-f_{j, t}\right),
\end{gathered}
$$

where $G_{j}(j=1,2, \ldots, m)$ is the available generating capacity that the unit $j$ of failure in the start-up state of $m$ units, while the other $(m-1)$ units are in normal state. The value of $p_{j}$ is the probability of the event $j$ occurrence, which can be described by

$$
\begin{gathered}
G_{j}=G_{0}-P_{j, \max }, \quad j=1,2, \ldots, m, \\
p_{j}=p_{0} \frac{f_{j, t}}{1-f_{j, t}}, \quad j=1,2, \ldots, m .
\end{gathered}
$$

Once the actual load of power system is greater than the available generating capacity, it would lead to part power outage. Therefore, the value of reliability index EENS at time $t E_{t}$ can be defined as

$$
E_{t}=\sum_{j=0}^{m} p_{j} \int_{G_{j}}^{+\infty} f(x)\left(x-G_{j}\right) d x
$$

\section{Solution to IRCUC Model Based on an Improved AFSA}

According to the above analysis, the IRCUC problem belongs to a class of NP-hard problems, as much, is very difficult to solve. Artificial fish swarm algorithm (AFSA), a novel intelligent algorithm, was first proposed in 2002 [20]. It has the potential to be one of the excellent techniques to obtain optimal or near-optimal solutions to realistic size IRCUC problems.

4.1. Artificial Fish Swarm Algorithm. In a water area, fish are most likely distributed around the region where foods are the most abundant. A fish swarm completes its food foraging process by each functioning several simple social behaviors. It is found that there are three most common fish behaviors: (1) searching behavior, that is, fish tend to head towards food; (2) swarming behavior, gregarious fish tend to concentrate towards each other while avoiding overcrowding; (3) following behavior, the behavior of chasing the nearest buddy. Inspired by swarm intelligence, AFSA is an artificial intelligent algorithm based on the simulation of collective behavior of real fish swarms. It simulates the behavior of a single artificial fish (AF) and then constructs a swarm of AF. Each AF will search its own local optimum, pass on information in its self-organized system, and finally achieve the global optimum.

Suppose the searching space is D-dimensional and there are $N$ fish in the colony. The current state of an AF is a vector $X=\left(x_{1}, x_{2}, \ldots, x_{n}\right)$, where $x_{i}(i=1,2, \ldots, n)$ is the variable to be optimized. The food consistence of AF in the current position is represented by $Y=f(X)$, where $Y$ is the objective function. The distance $D_{i j}$ between $X_{i}$ and $X_{j}$ of individual AF can be expressed as $\left\|X_{j}-X_{i}\right\| . \delta$ is defined as the factor of crowded degree, which represents the crowded degree of a location nearby and avoids more AF to gather together. Visual is the perception scope of an AF, which determines the moving direction of each AF. When the perception range of Visual becomes larger, the observation of an AF can be more comprehensive. However, the number of fish should grow more so as to reach the calculation amounts. Under the actual situation, the appropriate value should be selected by the specific case. Step is the largest moving step of an AF. For fear of missing the optimum solution, the length of Step should not be set too large. Of course, the length of Step is too small to converge. Try_number is represented as the number of the biggest trial in the searching behavior.

In the initial state of the algorithm, the variable of trial number should be defined as the trial times of AF searching for food. Then, the following steps describe the fish swarm behaviors.

(1) Searching Behavior. The fish follows the direction of food with high concentration when the fish is in the searching behavior. Suppose the current state of an $\mathrm{AF}$ is $X_{i}$ and its fitness is $Y_{i}$. A new state is randomly selected in its Visual field. If, in the maximum problem, $Y_{i}<Y_{j}$ (as the maximum problem and minimum problem can be converted with each other, the maximum problem is discussed as an example in the following analysis), move a step in that direction; otherwise, select a state randomly again and judge whether it satisfies the a forementioned condition. If it cannot be satisfied after predetermined times of Try_number, it moves a step randomly. The searching behavior is shown in Figure 1.

(2) Swarming Behavior. Swarming behavior is a behavior in which a fish swims towards to the central position of swarm and avoids overcrowding. An AF at current state $X_{i}$ seeks the companion's number $n_{f}$ and its central position in its current neighborhood $\left(D_{i j}<\right.$ Visual); if $Y_{c} / n_{f}>\delta Y_{i}$, this means that, at the center of the fish colony, there is enough food and it is not too crowded. The swarming behavior is illustrated in Figure 2.

(3) Following Behavior. Following behavior is the process where the fish captures the most active individual of swarm nearby. Suppose $X_{i}$ is the current state of AF searching companion $X_{\max }$ in the neighborhood with $Y_{\max }$; if $Y_{\max } / n_{f}>$ $\delta Y_{i}$, this means that the current position of companion $X_{\max }$ has a higher food consistence and it is not crowded enough. The AF will move a step towards the companion 


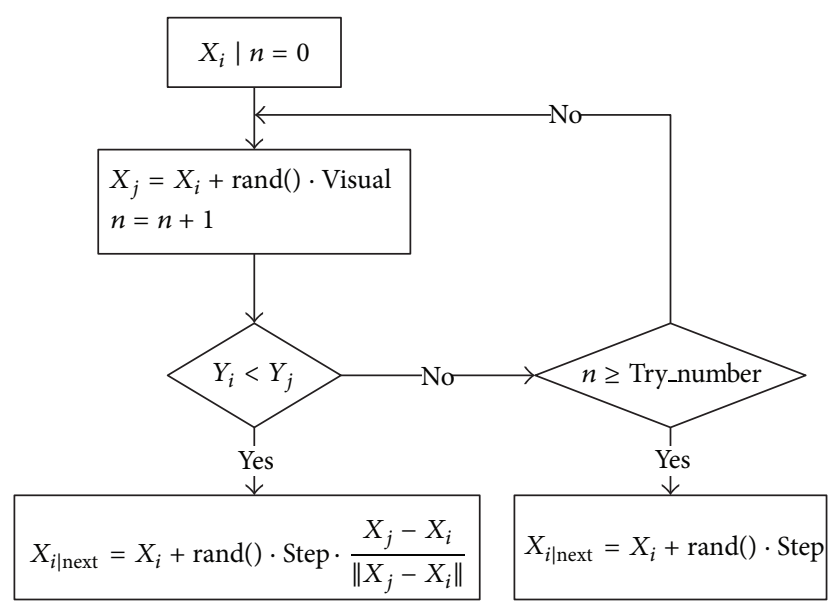

FIGURE 1: Searching behavior of AFSA.

$X_{\max }$; otherwise, it will continue the searching behavior. The following behavior can be seen in Figure 3 .

(4) Random Behavior. The realization of the random behavior is relatively simple. It means selecting a state randomly in the Visual field and swimming towards that direction. In fact, that is a default behavior of the searching behavior; namely, $X_{i \mid \text { next }}$ is the next position of $X_{i}$ :

$$
X_{i \mid \text { next }}=X_{i}+r \cdot \text { Visual, }
$$

where $r$ is the random number in $[-1,1]$.

(5) Bulletin. Bulletin is used to record the AF's optimal state and the optimal value of the problem. Each AF updates its own state and compares it with the bulletin after making movements. If its current state of $\mathrm{AF}$ is better, then the value on the bulletin will be replaced. At the end, the value on the bulletin is the optimal solution of the problem.

In the process of AFSA, searching behavior lays the foundation for the AF, swarming behavior enhances the convergence of stability, following behavior ensures the convergence of quickness, and behavior selection guarantees the high efficiency and stability of the algorithm. Through the behavior selection, the AFSA forms an optimization strategy with high efficiency.

4.2. AFSA with Added Mutation Operator. The AFSA has the ability to grasp the searching direction and avoid falling into the local optimum. But when some fish move in aimless random or gather around the local optima, the speed of convergence will be slowed down greatly and the searching accuracy is greatly reduced. To avoid premature convergence, an intelligent mutation operator (MO) similarly to genetic algorithm is introduced to enhance the ability escaping from the local optima in this paper. If the state of $\mathrm{AF}$ is not improved during the iterations and the $\mathrm{AF}$ has entered into a state of partial mining, it needs to be mutated. The specific steps are as follows.

(1) Randomly select one of the variables in the position to plus one, and choose the nonnull to minus one.

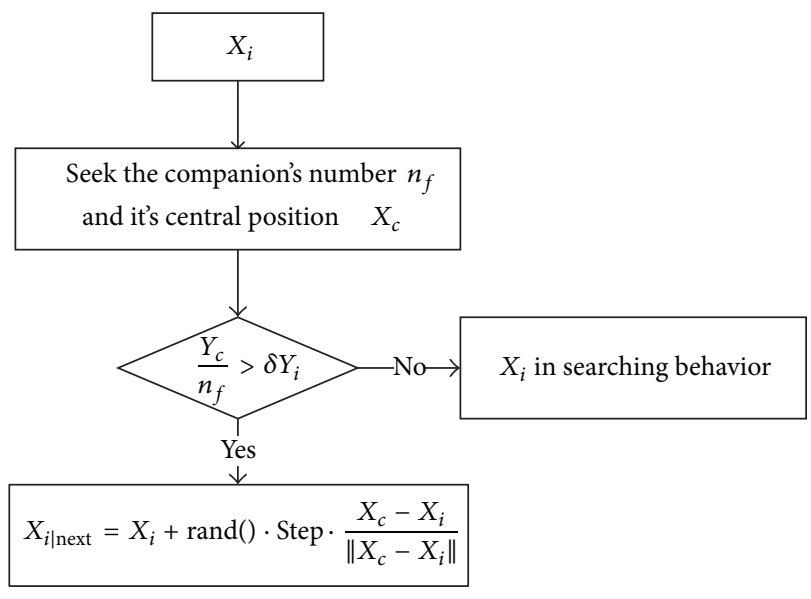

FIGURE 2: Swarming behavior of AFSA.

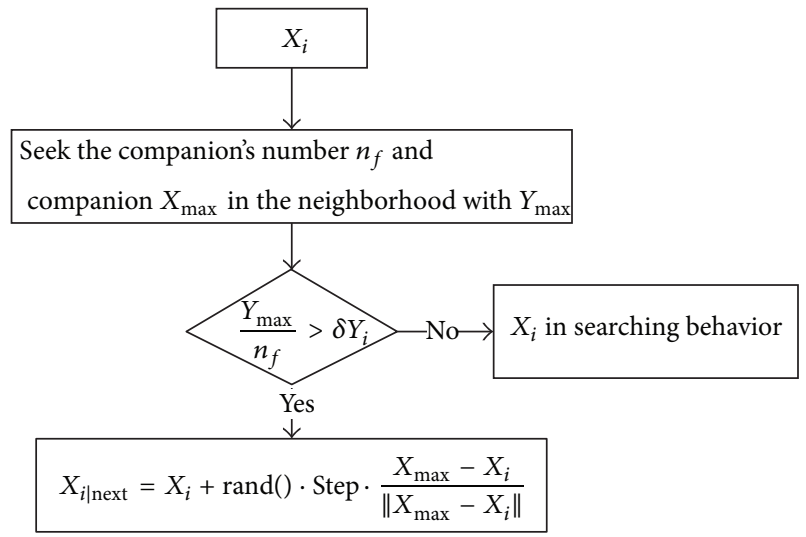

FIGURE 3: Following behavior of AFSA.

(2) If the value of state is better than that of the current state, update the state of position; otherwise, go back step (1) until the initial number of the mutating operation is satisfied.

By adding the mutation mechanism into the AFSA, it achieves the aim of altering the AF. Through adjusting the swarms, the rate of convergence and the global searching ability of AFSA are both improved. The selection of mutating probability will have a great influence on the performance of the proposed algorithm, which has a positive correlation to the elapsed time. According to the experimental experience, the probability of mutation operator (PMO) selected as $1 /(30 D) \sim 1 /(10 D)$ ( $D$ is the dimension) can obtain a good performance. Usually, the PMO of an AF is assumed as 0.03 0.1 .

4.3. IRCUC Model with Improved AFSA. In this paper, the binary coded matrix is used to denote the AF swarm. Each individual of $\mathrm{AF}$ swarm corresponds to a start-up planning of IRCUC model.

In this matrix, the element 0 refers to the outage state of the units; on the contrary, the element 1 corresponds to the power-on state. The AF in swarm is divided into two 
categories: the valid $\mathrm{AF}$ and the invalid AF. If the available generating capacity of AF is greater than the load forecasting at any time of the start-up planning, the AF is valid, or is otherwise invalid.

The food concentration of AF in current state is one of the important factors of AFSA, and its value determines the behavior selection in the optimization process. In the solving process of IRCUC model, economic dispatch (ED) should be computed for calculating the food concentration. The essence of ED is following the principle of the lowest fuel cost and assigns the power load to the state variables marked as 1 of units in the start-up planning. Considering that the ED calculation is time consuming, this algorithm only takes ED calculation by the valid AF. The invalid AF in the swarm is directly given poor food concentration and made to be sifted out gradually in the evolution process. After ED calculation, the total fuel cost of power generation is counted by the fuel cost function of each unit, which pluses the start-up cost of units to obtain the corresponding power generation cost of AF. It is needed to point out that the AF in accordance with the start-up planning may violate the constraints of the unit minimum uptime and downtime as mentioned in (6). Therefore, the penalty function algorithm is used to deal with the inequality constraints and to acquire the position concentration $V_{\text {fit }}$ ultimately; namely,

$$
V_{\text {fit }}=O_{f}\left(P_{i, t}, U_{i, t}\right)+\eta_{p} \times \beta \text {. }
$$

In (17), the first term is objective function of IRCUC model, $\eta_{p}$ is the predetermined large penalty coefficient, and $\beta$ is the number of times of violating the constraints of the unit minimum uptime and downtime.

When using the PL algorithm to solve the UC problem, the start-up order of each unit is determined by the average full-load cost of units. According to the start-up order, the MO operation should be adopted in this paper. The detailed descriptions are as follows.

Step 1. Compute the percentage of the value of reliability index $E_{t}$ in load forecasting $\delta_{t}$ at time $t$. If the value $\delta_{t}$ is greater than the predetermined upper threshold, this means the number of combination units is too small (that is, undersaturation); otherwise, if the value $\delta_{t}$ is less than the preset lower threshold, this means the number of combination units is too big (that is, oversaturation). Even though the outage loss is smaller at this time, it corresponds to the uneconomical start-up scheme.

Step 2. If it is undersaturation at that time, the outage units should take MO operation (start-up) according to the order of start-up, until the state of that time is not under-saturation.

Step 3. If it is over-saturation at that time, the operational units should take MO operation (closedown) according to the order of start-up, until the state of that time is not oversaturation. It is noticeable that the MO operation should be tried to avoid violating the constraints of the unit minimum uptime and downtime in (6) as far as possible.

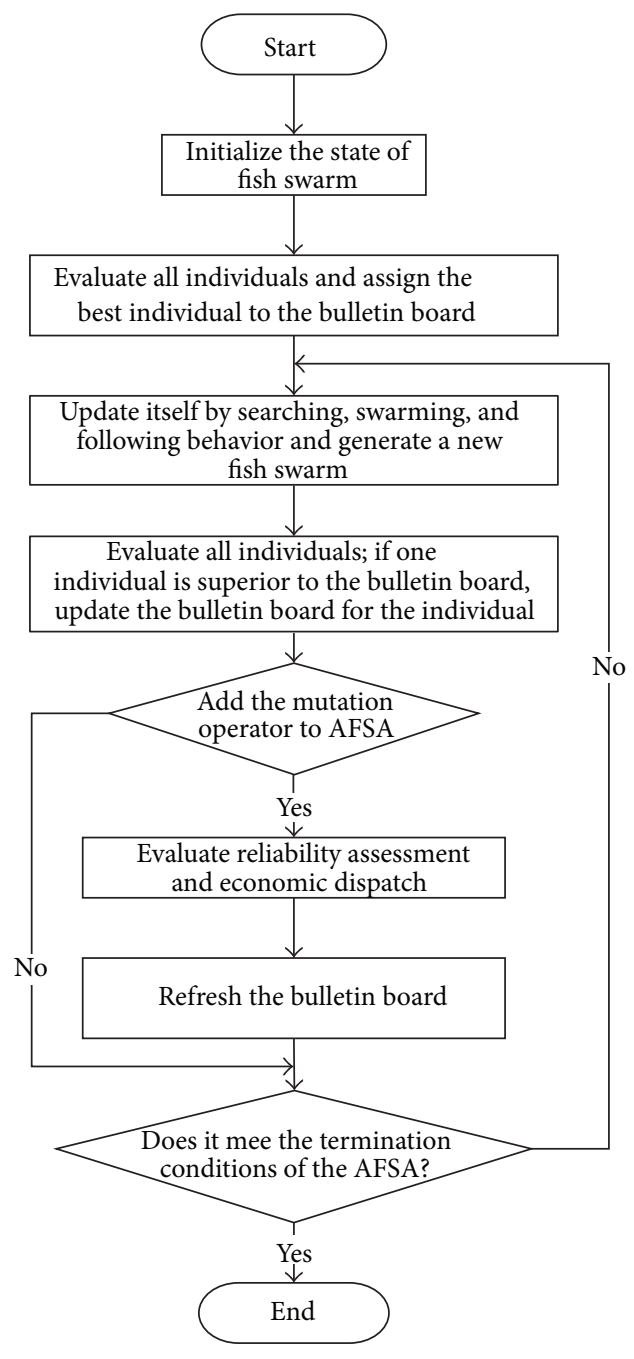

FIGURE 4: Flow chart of solving the IRCUC model with improved AFSA.

Note that the MO operation is executed in accordance with a certain probability which was mentioned in Section 4.2. In conclusion, the flow chart of solving the IRCUC model is shown in Figure 4.

\section{Numerical Analysis}

The efficiency of the proposed method is verified by solving the IRCUC model. The improved AFSA method is initially tested by systems with different sizes based on a basic system of 10 units from the literature [17-19]. The scheduling time horizon $T$ is chosen as one day with 24 intervals of one hour each.

5.1. Solution Quality and Convergence Characteristics. For assessing the reliability of the generation schedule, the reliability parameters of units are given in Table 1 and the standard deviation of random fluctuation of the load is accounted for $3 \%$ of the load forecasting. For estimating the outage cost 
TABLE 1: Reliability parameters of units.

\begin{tabular}{lc}
\hline Number of unit & Failure rate (times/hour) \\
\hline $1-2$ & 0.00033 \\
$3-5$ & 0.00050 \\
$6-7$ & 0.00151 \\
$8-10$ & 0.00075 \\
\hline
\end{tabular}

TABLE 2: Parameters of the proposed algorithm.

\begin{tabular}{lc}
\hline Parameter & Value \\
\hline Sizepop & 500 \\
Step & 0.3 \\
Visual & 1.8 \\
PMO & $0.03 \sim 0.1$ \\
Try_number & 10 \\
MAXGEN & 100 \\
Penalty coefficient $\eta_{P}$ & $10^{6}$ \\
Upper limit of $\delta_{t}$ & $0.5 \%$ \\
Lower limit of $\delta_{t}$ & $0.1 \%$ \\
$\delta$ & 0.618 \\
\hline
\end{tabular}

of the power system, the value of lost load is supposed as $1,000 \$ / \mathrm{MWh}$; namely, $V_{\mathrm{oll}}=1,000 \$ / \mathrm{MWh}$.

This paper uses the improved AFSA to schedule the planning of power generation and the output arrangement. The parameters of the proposed algorithm are shown in Table 2.

The solid line in Figure 5 shows the fitness function of the optimal location in the process of evolution. It can be seen from Figure 5 that this algorithm has a good convergence. The final result converges to the 37th generation and the corresponding total cost is $\$ 554,105$. In addition, the optimization result of AFSA without MO is also given in the dotted line of Figure 5 . The results show that the proposed MO can largely improve the optimization ability of the algorithm.

For comparison, this paper also uses the traditional UC model to optimize the planning of power generation and the scheduling of output power. In the process of optimization, the spinning reserve is assumed to be $5 \%$ and $10 \%$ of the load demand in each system. The results are illustrated in Figures 6 and 7 and Table 3.

The assessments of reliability under the optimal schedule of power generation are provided with each of the scheduling times in Figure 6. The reliability indexes of different cases are described in round, plus sign, and asterisk, respectively. Figure 7 points out the production cost and outage loss corresponding to the various optimization results. Table 3 shows the optimal scheduling of power generation under different situations. When the traditional UC model is used to optimize the generation schedule and to arrange the output power, the power generation schedule in Table 3 shows that the production cost and the outage loss are related to the predetermined spinning reserve requirements. In response to the increasing of spinning reserve, there will be more generating units in start-up state when the spinning reserves of system are gained from the $5 \%$ percentage of the load demand

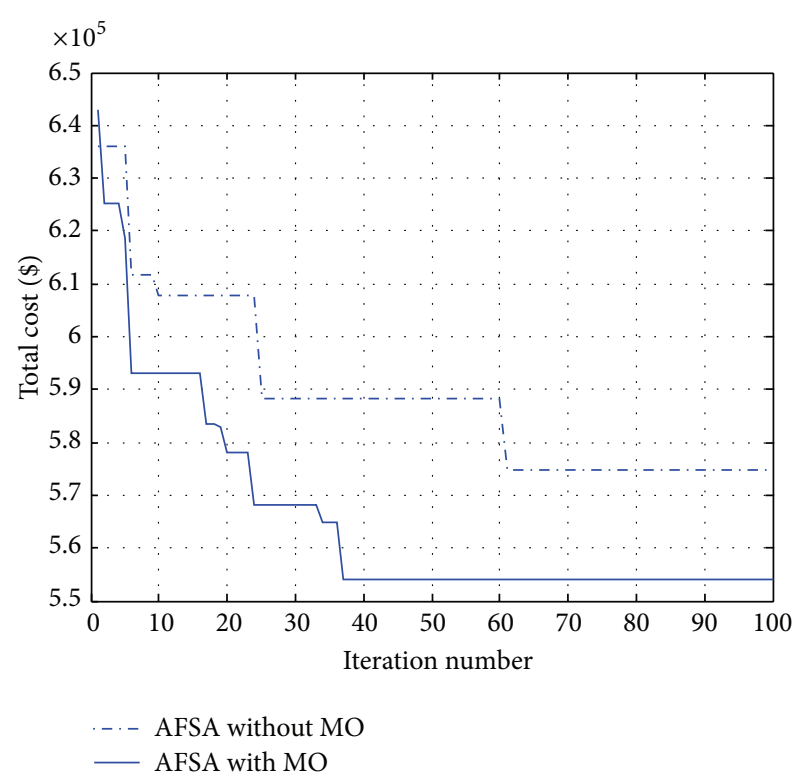

Figure 5: Total cost in the process of iterations.

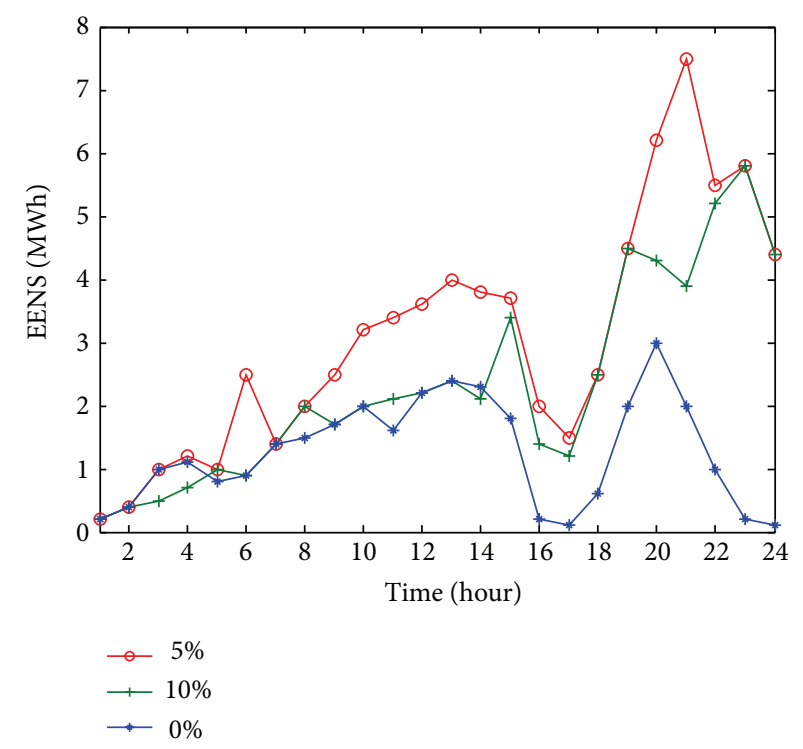

FIGURE 6: Reliability index of optimal generation schedule.

to $10 \%$ percentage. Therefore, the production cost increases from $\$ 509,165$ to $\$ 516,517$; on the contrary, the outage loss decreases from $\$ 65,425$ to $\$ 51,731$. Unlike the traditional UC model, the IRCUC model proposed in this paper contains an implicit reserve constraint. That is to say, the system spinning reserve would not be predetermined. The demand and reserve constraints imply in a tradeoff between the production cost and the outage loss. The calculation results show that more generating units of the power system would be scheduled to achieve the balance of the reliability and economy. Accordingly, the production cost further increases from $\$ 516,517$ to $\$ 526,921$, while the outage loss substantially further reduces from $\$ 51,731$ to $\$ 27,184$. Taking the effect of 
TABLE 3: Optimal scheduling of power generation (5\%, 10\%, and $0 \%$ of the load demand, resp.).

\begin{tabular}{|c|c|c|c|c|c|c|c|c|c|c|c|c|c|c|c|c|c|c|c|c|c|c|c|c|}
\hline \multirow{2}{*}{ Unit no. } & \multicolumn{24}{|c|}{ Hour } \\
\hline & 1 & 2 & 3 & 4 & 5 & 6 & 7 & 8 & 9 & 10 & 11 & 12 & 13 & 14 & 15 & 16 & 17 & 18 & 19 & 20 & 21 & 22 & 23 & 24 \\
\hline 1 & 1 & 1 & 1 & 1 & 1 & 1 & 1 & 1 & 1 & 1 & 1 & 1 & 1 & 1 & 1 & 1 & 1 & 1 & 1 & 1 & 1 & 1 & 1 & 1 \\
\hline 2 & 1 & 1 & 1 & 1 & 1 & 1 & 1 & 1 & 1 & 1 & 1 & 1 & 1 & 1 & 1 & 1 & 1 & 1 & 1 & 1 & 1 & 1 & 1 & 1 \\
\hline 3 & 0 & 0 & 0 & 1 & 1 & 1 & 1 & 1 & 1 & 1 & 1 & 1 & 1 & 1 & 1 & 1 & 1 & 1 & 1 & 1 & 0 & 0 & 0 & 0 \\
\hline 4 & 0 & 0 & 0 & 0 & 1 & 1 & 1 & 1 & 1 & 1 & 1 & 1 & 1 & 1 & 1 & 1 & 1 & 1 & 1 & 1 & 1 & 1 & 0 & 0 \\
\hline 5 & 0 & 0 & 0 & 0 & 0 & 1 & 1 & 1 & 1 & 1 & 1 & 1 & 1 & 1 & 1 & 1 & 1 & 1 & 1 & 1 & 1 & 1 & 0 & 0 \\
\hline 6 & 0 & 0 & 0 & 0 & 0 & 0 & 0 & 0 & 1 & 1 & 1 & 1 & 1 & 1 & 0 & 0 & 0 & 0 & 0 & 1 & 1 & 1 & 1 & 0 \\
\hline 7 & 0 & 0 & 0 & 0 & 0 & 0 & 0 & 0 & 1 & 1 & 1 & 0 & 0 & 0 & 0 & 0 & 0 & 0 & 0 & 1 & 1 & 1 & 0 & 0 \\
\hline 8 & 0 & 0 & 0 & 0 & 0 & 0 & 0 & 0 & 0 & 0 & 1 & 1 & 0 & 0 & 0 & 0 & 0 & 0 & 0 & 1 & 0 & 0 & 0 & 0 \\
\hline 9 & 0 & 0 & 0 & 0 & 0 & 0 & 0 & 0 & 0 & 0 & 0 & 1 & 0 & 0 & 0 & 0 & 0 & 0 & 0 & 0 & 0 & 0 & 0 & 0 \\
\hline 10 & 0 & 0 & 0 & 0 & 0 & 0 & 0 & 0 & 0 & 0 & 0 & 0 & 0 & 0 & 0 & 0 & 0 & 0 & 0 & 0 & 0 & 0 & 0 & 0 \\
\hline 1 & 1 & 1 & 1 & 1 & 1 & 1 & 1 & 1 & 1 & 1 & 1 & 1 & 1 & 1 & 1 & 1 & 1 & 1 & 1 & 1 & 1 & 1 & 1 & 1 \\
\hline 2 & 1 & 1 & 1 & 1 & 1 & 1 & 1 & 1 & 1 & 1 & 1 & 1 & 1 & 1 & 1 & 1 & 1 & 1 & 1 & 1 & 1 & 1 & 1 & 1 \\
\hline 3 & 0 & 0 & 1 & 1 & 1 & 1 & 1 & 1 & 1 & 1 & 1 & 1 & 1 & 1 & 1 & 1 & 1 & 1 & 1 & 1 & 1 & 0 & 0 & 0 \\
\hline 4 & 0 & 0 & 0 & 1 & 1 & 1 & 1 & 1 & 1 & 1 & 1 & 1 & 1 & 1 & 1 & 1 & 1 & 1 & 1 & 1 & 1 & 0 & 0 & 0 \\
\hline 5 & 0 & 0 & 0 & 0 & 0 & 1 & 1 & 1 & 1 & 1 & 1 & 1 & 1 & 1 & 1 & 1 & 1 & 1 & 1 & 1 & 1 & 1 & 0 & 0 \\
\hline 6 & 0 & 0 & 0 & 0 & 0 & 0 & 0 & 0 & 1 & 1 & 1 & 1 & 1 & 1 & 0 & 0 & 0 & 0 & 0 & 1 & 1 & 1 & 0 & 0 \\
\hline 7 & 0 & 0 & 0 & 0 & 0 & 0 & 0 & 0 & 1 & 1 & 1 & 1 & 1 & 1 & 0 & 0 & 0 & 0 & 0 & 1 & 1 & 1 & 1 & 1 \\
\hline 8 & 0 & 0 & 0 & 0 & 0 & 0 & 0 & 0 & 0 & 0 & 1 & 1 & 1 & 0 & 0 & 0 & 0 & 0 & 0 & 1 & 0 & 0 & 0 & 0 \\
\hline 9 & 0 & 0 & 0 & 0 & 0 & 0 & 0 & 0 & 0 & 0 & 1 & 1 & 0 & 0 & 0 & 0 & 0 & 0 & 0 & 0 & 0 & 0 & 0 & 0 \\
\hline 10 & 0 & 0 & 0 & 0 & 0 & 0 & 0 & 0 & 0 & 0 & 0 & 1 & 0 & 0 & 0 & 0 & 0 & 0 & 0 & 0 & 0 & 0 & 0 & 0 \\
\hline 1 & 1 & 1 & 1 & 1 & 1 & 1 & 1 & 1 & 1 & 1 & 1 & 1 & 1 & 1 & 1 & 1 & 1 & 1 & 1 & 1 & 1 & 1 & 1 & 1 \\
\hline 2 & 1 & 1 & 1 & 1 & 1 & 1 & 1 & 1 & 1 & 1 & 1 & 1 & 1 & 1 & 1 & 1 & 1 & 1 & 1 & 1 & 1 & 1 & 1 & 1 \\
\hline 3 & 0 & 0 & 0 & 0 & 0 & 1 & 1 & 1 & 1 & 1 & 1 & 1 & 1 & 1 & 1 & 1 & 1 & 1 & 1 & 1 & 1 & 1 & 1 & 1 \\
\hline 4 & 0 & 0 & 0 & 0 & 1 & 1 & 1 & 1 & 1 & 1 & 1 & 1 & 1 & 1 & 1 & 1 & 1 & 1 & 1 & 1 & 1 & 1 & 1 & 0 \\
\hline 5 & 0 & 0 & 0 & 1 & 1 & 1 & 1 & 1 & 1 & 1 & 1 & 1 & 1 & 1 & 1 & 1 & 1 & 1 & 1 & 1 & 1 & 1 & 1 & 1 \\
\hline 6 & 0 & 0 & 0 & 0 & 0 & 0 & 0 & 1 & 1 & 1 & 1 & 1 & 1 & 1 & 1 & 1 & 1 & 1 & 1 & 1 & 1 & 1 & 1 & 1 \\
\hline 7 & 0 & 0 & 0 & 0 & 0 & 0 & 0 & 0 & 1 & 1 & 1 & 1 & 1 & 1 & 1 & 1 & 1 & 1 & 1 & 1 & 1 & 1 & 0 & 0 \\
\hline 8 & 0 & 0 & 0 & 0 & 0 & 0 & 0 & 0 & 0 & 1 & 1 & 1 & 0 & 0 & 0 & 0 & 0 & 0 & 0 & 1 & 1 & 0 & 0 & 0 \\
\hline 9 & 0 & 0 & 0 & 0 & 0 & 0 & 0 & 0 & 0 & 0 & 1 & 1 & 0 & 0 & 0 & 0 & 0 & 0 & 0 & 0 & 0 & 0 & 0 & 0 \\
\hline 10 & 0 & 0 & 0 & 0 & 0 & 0 & 0 & 0 & 0 & 0 & 1 & 1 & 1 & 0 & 0 & 0 & 0 & 0 & 0 & 1 & 0 & 0 & 0 & 0 \\
\hline
\end{tabular}

the reliability into consideration, the better scheduling of power generation can be obtained by the IRCUC model.

Moreover, it should be emphasized that the IRCUC model is not needed to specify the capacity of spinning reserve in advance. Therefore, the IRCUC model is more practical and flexible than the normal UC model.

5.2. Effect of Optimization Results Caused by the Value of Lost Load. In the model of IRCUC, the value of $V_{\text {oll }}$ has a direct impact on the outage loss and further affects the final scheduling of power generation. To testify the influence of $V_{\text {oll }}$ on the optimization results, the value of $V_{\text {oll }}$ in 10 units system is assumed to be from $1,000 \$ / \mathrm{MWh}$ down to $500 \$ / \mathrm{MWh}$. At this time, Figure 8 and Table 4 show the production cost, and the EENS cost and the optimal scheduling of power generation which are optimized by the IRCUC model, respectively.

It can be seen from Figure 8 and Table 4 that the value of $V_{\text {oll }}$ has a significant effect on the optimization results. The decreasing of $V_{\text {oll }}$ signifies that the percentage of outage loss accounting for objective function is diminished. And the main goal of the IRCUC model in optimization scheduling of power generation focuses on reducing the production cost. Consequently, in order to meet the balance between the reliability and economy, the number of committed units in generation schedule is significantly dropped in Table 4. The production cost falls from $\$ 526,921$ to $\$ 516,467$ and the falling range is $\$ 10,454$. However, the EENS corresponding to the generation schedule increases from $27.18 \mathrm{MWh}$ to $40.74 \mathrm{MWh}$, the outage loss increases from $\$ 13,592$ to $\$ 20,370$, and the additional amount is only $\$ 6,778$.

5.3. Influence of Unit Number on Solution Quality and Computational Time. To verify the feasibility and effectiveness of the proposed method for solving large-scale IRCUC model, the simulations are tested on systems with 20,40,60, 80, and 100 units, respectively. For the systems with $20,40,60,80$, and 100 units, the basic 10 units system is duplicated and the total production costs are adjusted proportionally to the system sizes. To avoid any hazardous interpretation of optimization 
TABLE 4: Scheduling of power generation with different $V_{\text {oll }}(1,000 \$ / \mathrm{MWh}$ and 500\$/MWh, resp.).

\begin{tabular}{|c|c|c|c|c|c|c|c|c|c|c|c|c|c|c|c|c|c|c|c|c|c|c|c|c|}
\hline \multirow{2}{*}{ Unit no. } & \multicolumn{24}{|c|}{ Hour } \\
\hline & 1 & 2 & 3 & 4 & 5 & 6 & 7 & 8 & 9 & 10 & 11 & 12 & 13 & 14 & 15 & 16 & 17 & 18 & 19 & 20 & 21 & 22 & 23 & 24 \\
\hline 1 & 1 & 1 & 1 & 1 & 1 & 1 & 1 & 1 & 1 & 1 & 1 & 1 & 1 & 1 & 1 & 1 & 1 & 1 & 1 & 1 & 1 & 1 & 1 & 1 \\
\hline 2 & 1 & 1 & 1 & 1 & 1 & 1 & 1 & 1 & 1 & 1 & 1 & 1 & 1 & 1 & 1 & 1 & 1 & 1 & 1 & 1 & 1 & 1 & 1 & 1 \\
\hline 3 & 0 & 0 & 0 & 1 & 1 & 1 & 1 & 1 & 1 & 1 & 1 & 1 & 1 & 1 & 1 & 1 & 1 & 1 & 1 & 1 & 1 & 1 & 1 & 0 \\
\hline 4 & 0 & 0 & 0 & 0 & 1 & 1 & 1 & 1 & 1 & 1 & 1 & 1 & 1 & 1 & 1 & 1 & 1 & 1 & 1 & 1 & 1 & 1 & 1 & 1 \\
\hline 5 & 0 & 0 & 0 & 0 & 1 & 1 & 1 & 1 & 1 & 1 & 1 & 1 & 1 & 1 & 1 & 1 & 1 & 1 & 1 & 1 & 1 & 1 & 1 & 1 \\
\hline 6 & 0 & 0 & 0 & 0 & 0 & 0 & 0 & 0 & 1 & 1 & 1 & 1 & 1 & 1 & 1 & 1 & 1 & 1 & 1 & 1 & 1 & 1 & 0 & 0 \\
\hline 7 & 0 & 0 & 0 & 0 & 0 & 0 & 0 & 0 & 0 & 1 & 1 & 1 & 1 & 1 & 0 & 0 & 0 & 0 & 0 & 1 & 1 & 0 & 0 & 0 \\
\hline 8 & 0 & 0 & 0 & 0 & 0 & 0 & 0 & 0 & 0 & 0 & 1 & 1 & 1 & 0 & 0 & 0 & 0 & 0 & 0 & 0 & 0 & 0 & 0 & 0 \\
\hline 9 & 0 & 0 & 0 & 0 & 0 & 0 & 0 & 0 & 0 & 0 & 1 & 1 & 0 & 0 & 0 & 0 & 0 & 0 & 0 & 0 & 0 & 0 & 0 & 0 \\
\hline 10 & 0 & 0 & 0 & 0 & 0 & 0 & 0 & 0 & 0 & 0 & 0 & 0 & 0 & 0 & 0 & 0 & 0 & 0 & 0 & 1 & 0 & 0 & 0 & 0 \\
\hline 1 & 1 & 1 & 1 & 1 & 1 & 1 & 1 & 1 & 1 & 1 & 1 & 1 & 1 & 1 & 1 & 1 & 1 & 1 & 1 & 1 & 1 & 1 & 1 & 1 \\
\hline 2 & 1 & 1 & 1 & 1 & 1 & 1 & 1 & 1 & 1 & 1 & 1 & 1 & 1 & 1 & 1 & 1 & 1 & 1 & 1 & 1 & 1 & 1 & 1 & 1 \\
\hline 3 & 0 & 0 & 0 & 0 & 0 & 1 & 1 & 1 & 1 & 1 & 1 & 1 & 1 & 1 & 1 & 1 & 1 & 1 & 1 & 1 & 1 & 1 & 1 & 1 \\
\hline 4 & 0 & 0 & 0 & 0 & 1 & 1 & 1 & 1 & 1 & 1 & 1 & 1 & 1 & 1 & 1 & 1 & 1 & 1 & 1 & 1 & 1 & 1 & 1 & 0 \\
\hline 5 & 0 & 0 & 0 & 1 & 1 & 1 & 1 & 1 & 1 & 1 & 1 & 1 & 1 & 1 & 1 & 1 & 1 & 1 & 1 & 1 & 1 & 1 & 1 & 1 \\
\hline 6 & 0 & 0 & 0 & 0 & 0 & 0 & 0 & 0 & 1 & 1 & 1 & 1 & 1 & 1 & 1 & 1 & 1 & 1 & 1 & 1 & 1 & 1 & 1 & 1 \\
\hline 7 & 0 & 0 & 0 & 0 & 0 & 0 & 0 & 0 & 1 & 1 & 1 & 1 & 1 & 1 & 1 & 1 & 1 & 1 & 1 & 1 & 1 & 1 & 0 & 0 \\
\hline 8 & 0 & 0 & 0 & 0 & 0 & 0 & 0 & 0 & 0 & 1 & 1 & 1 & 0 & 0 & 0 & 0 & 0 & 0 & 0 & 1 & 1 & 0 & 0 & 0 \\
\hline 9 & 0 & 0 & 0 & 0 & 0 & 0 & 0 & 0 & 0 & 0 & 1 & 1 & 0 & 0 & 0 & 0 & 0 & 0 & 0 & 0 & 0 & 0 & 0 & 0 \\
\hline 10 & 0 & 0 & 0 & 0 & 0 & 0 & 0 & 0 & 0 & 0 & 1 & 1 & 1 & 0 & 0 & 0 & 0 & 0 & 0 & 1 & 0 & 0 & 0 & 0 \\
\hline
\end{tabular}

TABLE 5: Comparison of the total production costs (\$) for up to 100 units using IRCUC model with other methods $\left(V_{\text {oll }}=1,000 \$ / \mathrm{MWh}\right)$.

\begin{tabular}{|c|c|c|c|c|c|c|c|c|}
\hline \multirow{2}{*}{ Units } & \multicolumn{2}{|c|}{ GA } & \multicolumn{2}{|c|}{ PSO } & \multicolumn{2}{|c|}{$\mathrm{ACO}$} & \multicolumn{2}{|c|}{ IAFSA } \\
\hline & Best & Worst & Best & Worst & Best & Worst & Best & Worst \\
\hline 10 & 554,943 & 556,121 & 555,404 & 556,231 & 556,686 & 558,006 & 554,105 & 554,579 \\
\hline 20 & $1,110,068$ & $1,125,790$ & $1,119,244$ & $1,133,793$ & $1,112,292$ & $1,117,054$ & $1,108,979$ & $1,110,643$ \\
\hline 40 & $2,219,103$ & $2,229,024$ & $2,219,523$ & $2,240,085$ & $2,220,116$ & $2,239,146$ & $2,218,163$ & $2,223,117$ \\
\hline 60 & $3,340,066$ & $3,352,886$ & $3,342,108$ & $3,359,012$ & $3,340,407$ & $3,353,921$ & $3,338,979$ & $3,343,125$ \\
\hline 80 & $4,460,202$ & $4,472,478$ & $4,465,349$ & $4,480,939$ & $4,465,177$ & $4,477,753$ & $4,457,032$ & $4,461,943$ \\
\hline 100 & $5,583,977$ & $5,658,925$ & $5,617,388$ & $5,680,841$ & $5,590,607$ & $5,651,827$ & $5,581,284$ & $5,608,291$ \\
\hline
\end{tabular}

results, related to the choice of particular initial states, 10 trials for each units system are performed. The best and worst total production costs of the improved AFSA (IAFSA) are obtained with different $V_{\text {oll }}$ in Tables 5 and 6, respectively.

As shown in Tables 5 and 6, the average production costs of 10 trials using the improved AFSA generated variation in a small range and the standard deviations are small and tolerable. It is demonstrated that the improved AFSA method has a better quality of solution and robustness for the IRCUC model. To validate the results obtained with the proposed method, we compare the performance of the improved AFSA to those of other approaches with respect to the total production costs. Tables 5 and 6 provide the comparison of the total production costs from the improved AFSA method to those of other methods with different value of $V_{\text {oll }}$. It is clearly shown that the total production costs by the improved AFSA for large-scale systems constantly outperform those obtained by GA, PSO, and ACO. Meanwhile, it is obvious that the value of $V_{\text {oll }}$ still plays an important role in the optimization of the power generation. Considering more information of AF to control the mutation operation, the proposed method can find the best solution. Therefore, the improved AFSA has the potential applications to the real system.

In addition, the computational times of the improved AFSA and other methods are another important evaluation index. The computational times of the above methods to find the optimal solutions with various numbers of units to be committed are shown in Table 7. Analysis of the results presented in Table 7 shows that the computational time of the improved AFSA is in direct proportion to the number of units and the system sizes. From Table 7, it is clear that the computational efficiency of the improved AFSA has been improved significantly compared with that of other methods. All these situations have shown that the improved AFSA method provides excellent performances: fast computation speed, stable convergence, and cost saving system. Moreover, the computational times of the improved AFSA are not 
TABLE 6: Comparison of the total production costs (\$) for up to 100 units using IRCUC model with other methods $\left(V_{\text {oll }}=500 \$ / M W h\right)$.

\begin{tabular}{|c|c|c|c|c|c|c|c|c|}
\hline \multirow{2}{*}{ Units } & \multicolumn{2}{|c|}{ GA } & \multicolumn{2}{|c|}{ PSO } & \multicolumn{2}{|c|}{$\mathrm{ACO}$} & \multicolumn{2}{|c|}{ IAFSA } \\
\hline & Best & Worst & Best & Worst & Best & Worst & Best & Worst \\
\hline 10 & 537,582 & 540,312 & 537,515 & 549,530 & 538,797 & 546,660 & 536,837 & 537,279 \\
\hline 20 & $1,076,156$ & $1,085,332$ & $1,088,494$ & $1,102,101$ & $1,078,919$ & $1,090,458$ & $1,075,279$ & $1,079,034$ \\
\hline 40 & $2,152,751$ & $2,168,248$ & $2,159,903$ & $2,180,850$ & $2,160,012$ & $2,174,762$ & $2,148,968$ & $2,155,008$ \\
\hline 60 & $3,238,565$ & $3,259,686$ & $3,240,161$ & $3,278,542$ & $3,245,471$ & $3,269,129$ & $3,232,139$ & $3,240,654$ \\
\hline 80 & $4,388,004$ & $4,426,215$ & $4,395,974$ & $4,438,793$ & $4,395,848$ & $4,434,752$ & $4,381,521$ & $4,388,022$ \\
\hline 100 & $5,428,795$ & $5,479,510$ & $5,439,588$ & $5,492,534$ & $5,430,955$ & $5,485,750$ & $5,407,844$ & $5,429,125$ \\
\hline
\end{tabular}

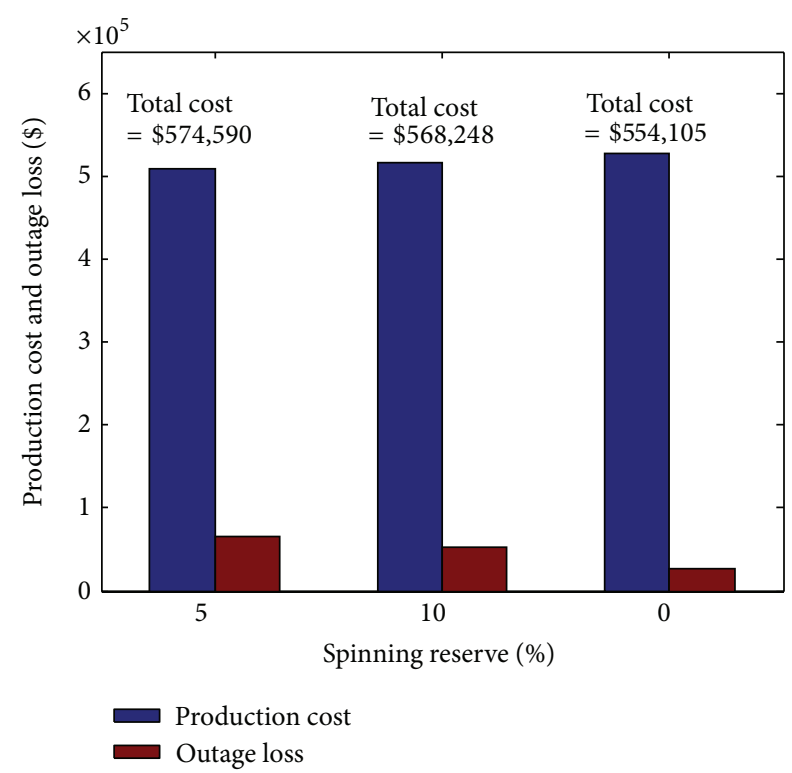

Figure 7: Production cost and outage loss.

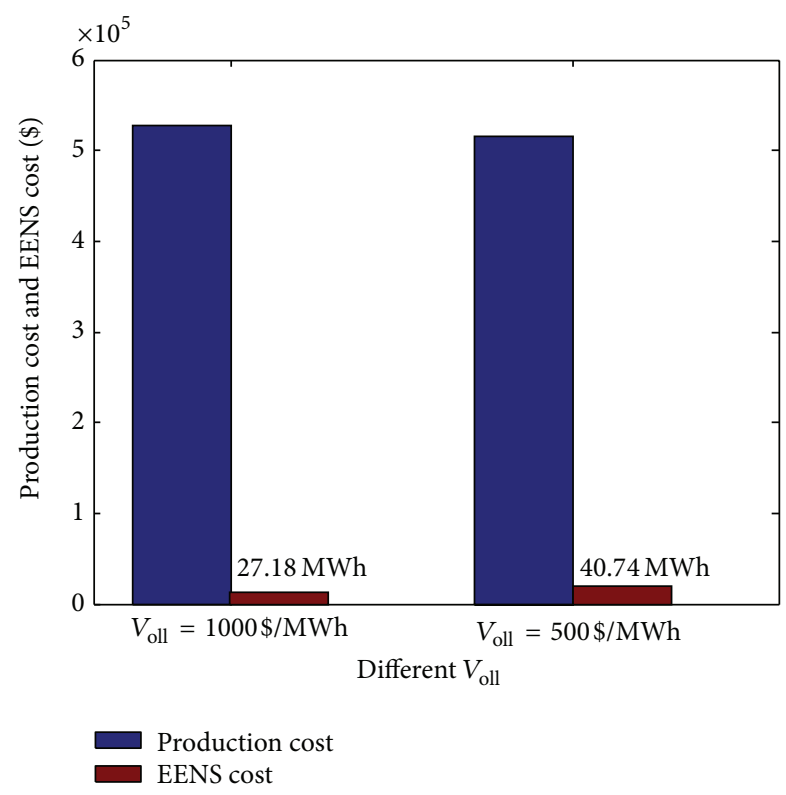

FIGURE 8: Production cost and EENS cost with different $V_{\text {oll }}$.
TABle 7: Comparison of the computational times (s) for up to 100 units using IRCUC model with other methods.

\begin{tabular}{lcccc}
\hline \multirow{2}{*}{ Units } & \multicolumn{4}{c}{ Computational times } \\
& GA & PSO & ACO & IAFSA \\
\hline 10 & 90 & 55 & 25 & 19 \\
20 & 255 & 158 & 60 & 45 \\
40 & 728 & 440 & 180 & 95 \\
60 & 995 & 885 & 355 & 182 \\
80 & 1,780 & 1,624 & 595 & 242 \\
100 & 3,310 & 2,825 & 860 & 448 \\
\hline
\end{tabular}

increasing exponentially with respect to the system size of IRCUC model, which is favorable for the large-scale system. By the way, it is noticed that the increasing rate of the computational times of the improved AFSA is polynomial to the problem size.

It follows from Table 5 to Table 7 that the total production costs of the improved AFSA are less expensive than those obtained by using other methods on all of the generating systems. Obviously, the improved AFSA improves performance vastly more than other methods in terms of both solution quality and computational time especially on the large-scale implementation. The proposed method converges to the solution at a faster rate than the above-mentioned methods.

\section{Conclusions}

To overcome the deficiency of UC model, this paper tried to remove the constraint of spinning reserve from the traditional UC model and the IRCUC model was proposed. The objective function was extended from the minimal production cost of traditional UC model to the minimal sum of the production cost and the outage loss. The demand and reserve constraints were not explicitly given in the IRCUC model. Rather, the tradeoff between the production cost and the outage loss of the objective function was implicit.

The IRCUC model was solved by using the AFSA. In addition to the standard operation of AFSA, the MO operation was designed with the idea of PL algorithm and dramatically improved the optimizing ability of AFSA.

The efficiency of the proposed method was demonstrated by using the testing systems with the number of generating units from 10 to 100 . The numerical results showed 
the improvements in effectiveness and computational time compared to the results obtained from other methods. Furthermore, the results proved that the method is capable of solving realistic UC problems.

\section{Acknowledgments}

This work is supported by the Graduate Education Innovation Project in Jiangsu Province (no. CXZZ12_0228). The authors would like to thank the editor and anonymous reviewers for their suggestions in improving the quality of the paper.

\section{References}

[1] I. G. Damousis, A. G. Bakirtzis, and P. S. Dokopoulos, "A solution to the unit-commitment problem using integer-coded genetic algorithm," IEEE Transactions on Power Systems, vol. 19, no. 2, pp. 1165-1172, 2004.

[2] M. M. Hadji and B. Vahidi, "A solution to the unit commitment problem using imperialistic competition algorithm," IEEE Transactions on Power Systems, vol. 27, no. 1, pp. 117-124, 2012.

[3] M. A. Ortega-Vazquez and D. S. Kirschen, "Estimating the spinning reserve requirements in systems with significant wind power generation penetration," IEEE Transactions on Power Systems, vol. 24, no. 1, pp. 114-124, 2009.

[4] L. T. Anstine, R. E. Burke, J. E. Casey, R. Holgate, R. S. John, and H. G. Stewart, "Application of probability methods to the determination of spinning reserve requirements for the Pennsylvania-New Jersey-Maryland interconnection," IEEE Transactions on Power Apparatus and Systems, vol. 82, no. 68, pp. 726-735, 1963.

[5] W. Y. Li and J. Q. Zhou, "Probabilistic reliability assessment of power system operations," Electric Power Components and Systems, vol. 36, no. 10, pp. 1102-1114, 2008.

[6] W. L. Snyder, H. D. Powell, and J. C. Rayburn, "Dynamic programming approach to unit commitment," IEEE Transactions on Power Systems, vol. 2, no. 2, pp. 339-350, 1987.

[7] R. C. Johnson, H. H. Happ, and W. J. Wright, "Large scale hydro-thermal unit commitment-method and results," IEEE Transactions on Power Apparatus and Systems, vol. 90, no. 3, pp. 1373-1384, 1971.

[8] T. Senjyu, K. Shimabukuro, K. Uezato, and T. Funabashi, "A fast technique for unit commitment problem by extended priority list," IEEE Transactions on Power Systems, vol. 18, no. 2, pp. 882888, 2003.

[9] E. C. Finardi and E. L. da Silva, "Solving the hydro unit commitment problem via dual decomposition and sequential quadratic programming," IEEE Transactions on Power Systems, vol. 21, no. 2, pp. 835-844, 2006.

[10] H. L. Ma and S. M. Shahidehpour, "Transmission-constrained unit commitment based on Benders decomposition," International Journal of Electrical Power and Energy Systems, vol. 20, no. 4, pp. 287-294, 1998.

[11] C. P. Cheng and C. W. Liu, "Unit commitment by lagrangian relaxation and genetic algorithms," IEEE Transactions on Power Systems, vol. 15, no. 2, pp. 707-714, 2000.

[12] W. Ongsakul and N. Petcharaks, "Unit commitment by enhanced adaptive lagrangian relaxation," IEEE Transactions on Power Systems, vol. 19, no. 1, pp. 620-628, 2004.

[13] A. Frangioni, C. Gentile, and F. Lacalandra, "Tighter approximated MILP formulations for unit commitment problems,"
IEEE Transactions on Power Systems, vol. 24, no. 1, pp. 105-113, 2009.

[14] K. S. Swarup and S. Yamashiro, "Unit commitment solution methodology using genetic algorithm," IEEE Transactions on Power Systems, vol. 17, no. 1, pp. 87-91, 2002.

[15] T. Seniyu, A. Y. Saber, T. Miyagi, K. Shimabukuro, N. Urasaki, and T. Funabashi, "Fast technique for unit commitment by genetic algorithm based on unit clustering," IEE ProceedingGeneration Transmission and Distribution, vol. 152, no. 5, pp. 705-713, 2005.

[16] J. M. Arroyo and A. J. Conejo, "A parallel repair genetic algorithm to solve the unit commitment problem," IEEE Transactions on Power Systems, vol. 17, no. 4, pp. 1216-1224, 2002.

[17] I. J. Raglend, C. Raghuveer, G. R. Avinash, N. P. Padhy, and D. P. Kothari, "Solution to profit based unit commitment problem using particle swarm optimization," Applied Soft Computing Journal, vol. 10, no. 4, pp. 1247-1256, 2010.

[18] J. Hao, L. B. Shi, and J. Q. Zhou, "Optimal unit commitment based on ant colony optimization algorithm," Power System Technology, vol. 26, no. 1, pp. 26-31, 2002.

[19] D. N. Simopoulos, S. D. Kavatza, and C. D. Vournas, "Reliability constrained unit commitment using simulated annealing," IEEE Transactions on Power Systems, vol. 21, no. 4, pp. 1699-1706, 2006.

[20] X. L. Li, A New Intelligent Optimization Method-Artificial Fish Swarm Algorithm, Zhejiang University, Hangzhou, China, 2003.

[21] X. L. Li, F. Lu, and G. H. Tian, "Applications of artificial fish school algorithm in combinatorial optimization problem," Journal of Shandong University, vol. 34, pp. 64-67, 2004.

[22] L. G. Wang and Q. H. Song, "Parameters analysis of artificial fish swarm algorithm," Computer Engineering, no. 24, pp. 169$171,2010$.

[23] X. G. Yang, Y. Q. Zhang, and Y. M. Li, "Hybrid algorithm with artificial fish swarm algorithm and PSO," Application Research of Computers, no. 6, pp. 2082-2087, 2010.

[24] H. M. Jiang, K. Xie, and Y. F. Wang, "Optimization of pump parameters for gain flattened Raman fiber amplifiers based on artificial fish school algorithm," Optics Communications, vol. 284, no. 23, pp. 5480-5483, 2011.

[25] W. L. Zhu, J. Q. Jiang, C. Y. Song, and L. Y. Bao, "Clustering algorithm based on fuzzy C-means and artificial fish swarm," Procedia Engineering, vol. 29, pp. 3307-3311, 2012.

[26] F. Vallée, J. Lobry, and O. Deblecker, "System reliability assessment method for wind power integration," IEEE Transactions on Power Systems, vol. 23, no. 3, pp. 1288-1297, 2008.

[27] R. Billinton, B. Bagen, and Y. Cui, "Reliability evaluation of small stand-alone wind energy conversion systems using a time series simulation model," IEE Proceedings-Generation, Transmission and Distribution, vol. 150, no. 1, pp. 96-100, 2003. 


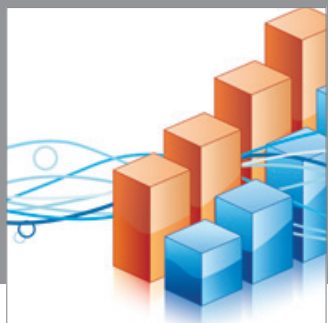

Advances in

Operations Research

mansans

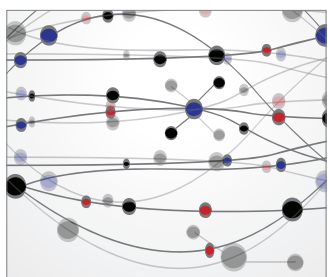

The Scientific World Journal
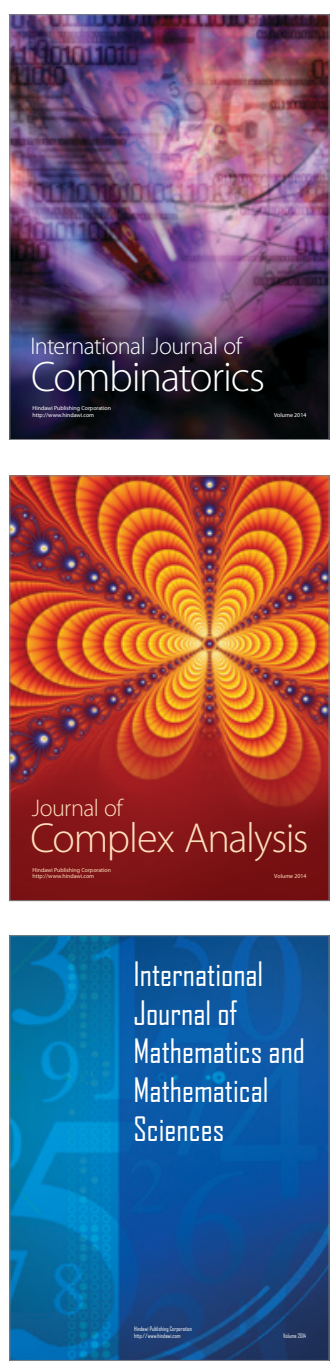
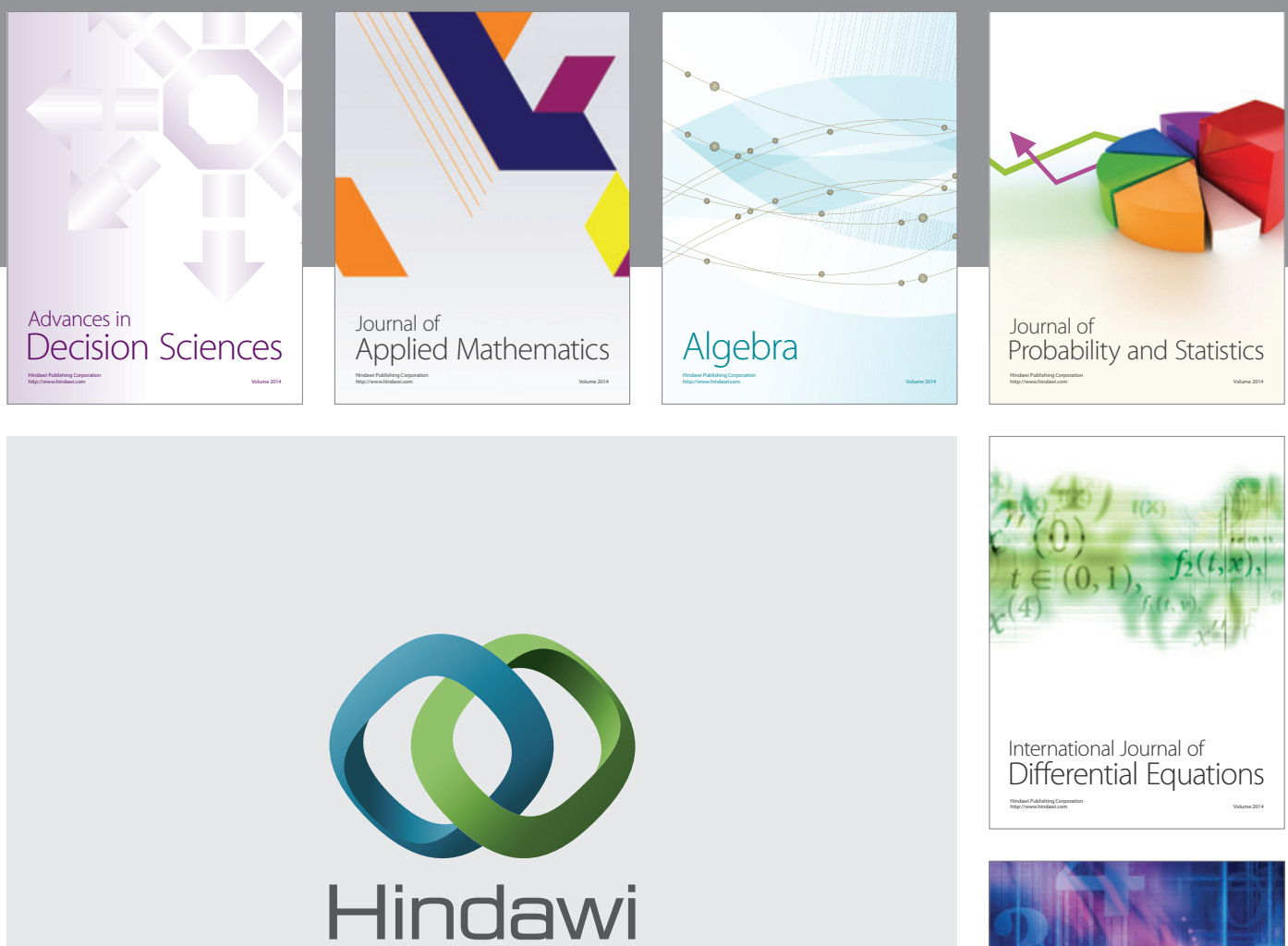

Submit your manuscripts at http://www.hindawi.com
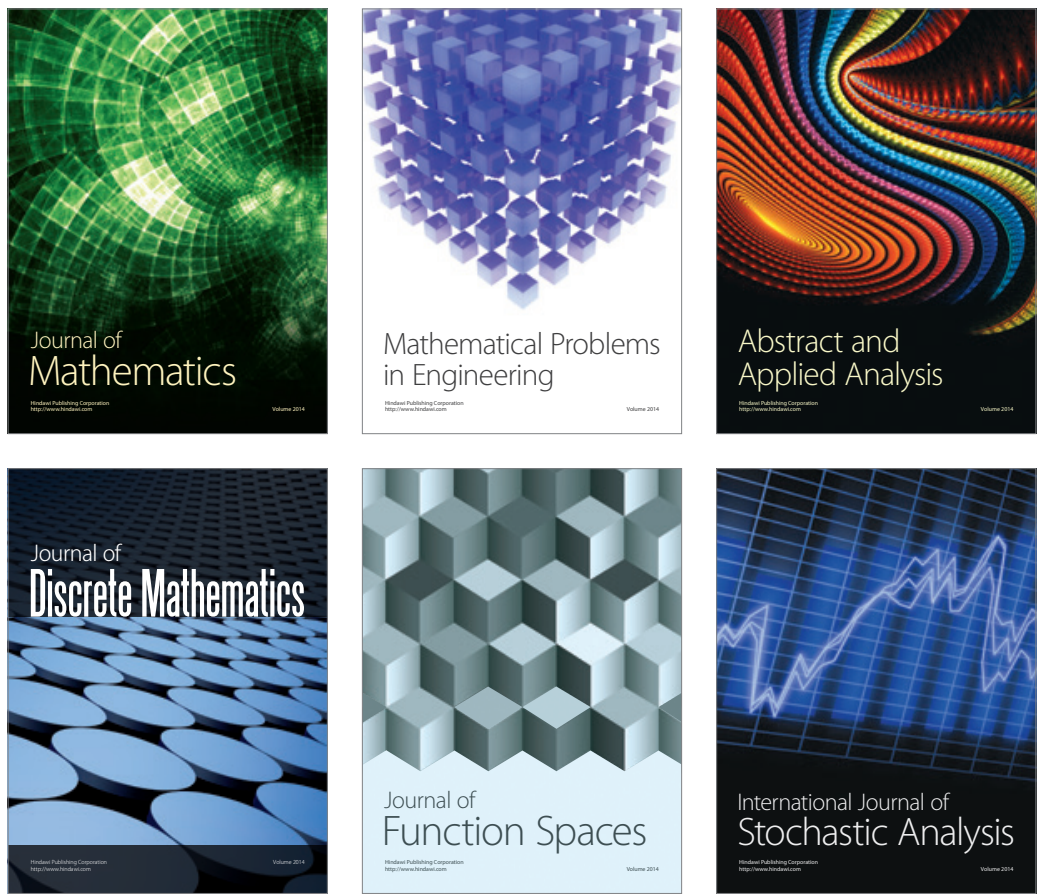

Journal of

Function Spaces

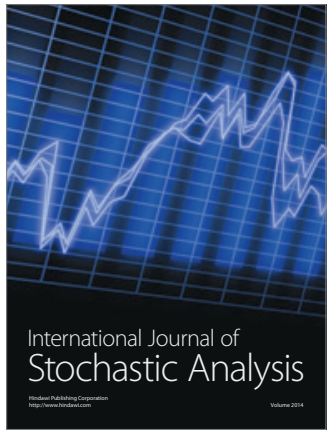

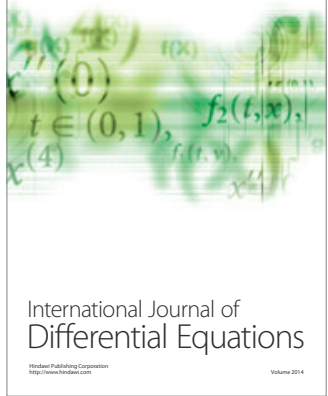
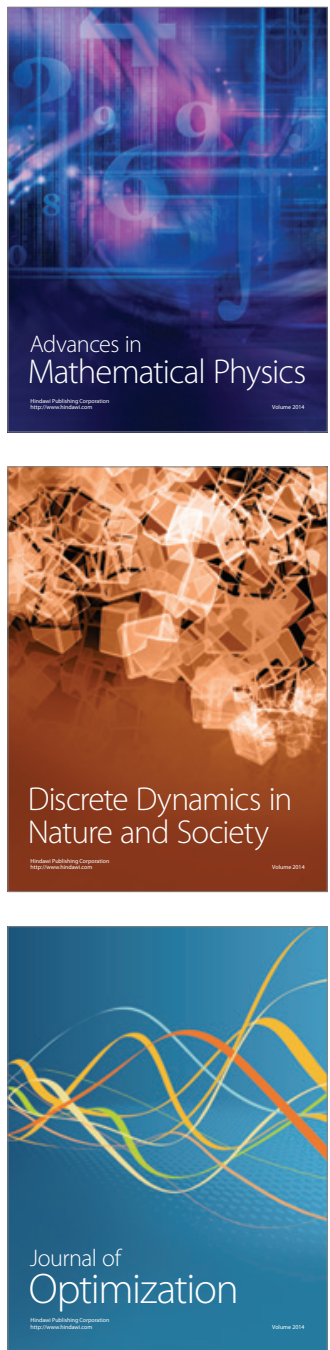